\title{
P02-4-5 Poster session
}

\section{The dysfunction of clock genes induces nocturia associated with disrupted gene expression rhythm of mechano-sensory channels in the mouse bladder urothelium}

\author{
Tatsuya Ihara, Masayoshi Tanaka, Eiji Shigetomi, Yoichi Shinozaki, Shuichi Koizumi
}

Department of Neuropharmacology, University of Yamanashi, Japan

Backgrounds

The etiology of nocturia is multifactorial and remains unclear in a large number of patients. Clock genes exist in most cells and organs, and the products of clock genes create circadian rhythm in sleep-awake rhythms and the gene expression of various metabolic enzymes, channels, and receptors. It has been reported that clock genes regulate lower urinary tract function, and a new concept is suggested that abnormalities of clock genes may cause lower urinary tract symptoms such as nocturia. On the other hand, the bladder urothelium can senses bladder wall extension via mechano-sensory channels such as TRPV4 and Piezo1, then transmit the signal of urine sensation. We hypothesized that expression rhythm in mechano-sensory channels were regulated by clock genes with circadian rhythm, and nocturia was induced by dysfunction of clock genes, which caused the abnormal gene expression rhythm of mechano-sensory channels and urine sensation in the bladder urothelium. In this study, we investigated voiding behavior and gene expression rhythm of clock genes and mechano-sensory channels in the mouse bladder urothelium to reveal the effect of clock genes on nocturia.

Methods

MaleC57BL/6 mice (WT) and Clock mutant $\left(\right.$ Clock $\left.^{\Delta 19 / \Delta 19}\right)$ mice were used. They were bred under 12-h light/dark conditions for 2 weeks and voiding behavior was measured using metabolic cages. Bladder was excised every 4 hours, and then bladder urothelium was isolated. The gene expression rhythm in clock genes and mechano-sensory channels were quantified in the bladder urothelium using RT-PCR.

Results

Clock $^{419 / \Delta 19}$ mice showed higher voiding frequencies during the sleep phase than WT mice. The diurnal change in urine volume/voiding between the dark and light, which observed in WT mice, was absent in Clock ${ }^{19 / \Delta 19}$ mice. Additionally, functional bladder capacity was significantly lower in Clock $^{\Delta 19 / \Delta 19}$ mice than in WT mice.

WT mice showed circadian gene expression in clock genes and mechano-sensory channels. However, Clock ${ }^{\Delta 19 / \Delta 19}$ mice lost circadian gene expression not only in clock genes but also mechano-sensory channels.

Conclusions

We demonstrated that $C_{l o c k}^{\Delta 19 / \Delta 19}$ mice showed the phenotype of nocturia. The loss of circadian urine sensation due to the abnormalities in clock genes may be one of the causes of nocturia. 